\title{
Explosion-induced damage to oilwell perforating gun carriers
}

\author{
B. Grove, A. Werner \& C. Han \\ Schlumberger Oilfield Services
}

\begin{abstract}
We present an overview of damage to oilwell perforating gun carrier tubes. Gun carriers are sealed hollow steel tubes, used to convey multiple small shaped explosive charges down a wellbore to the vicinity of a hydrocarbon reservoir, isolating the charges from the wellbore fluid and pressure. At the prescribed time and location the charges are detonated, producing perforation tunnels into the reservoir and allowing subsequent hydrocarbon flow into the wellbore and uphole to surface facilities.

Upon detonation, the charges present three significant and damaging loading mechanisms within the carrier tube: jet perforation, case fragment impact, and explosive blast. The resulting carrier tube has several holes and bulges, but is otherwise intact; it is then either retrieved to surface or dropped into the rathole.

It is generally desired that a gun carrier tube survives the perforation event without excessive swelling, cracking, or catastrophic rupturing (i.e. splitting). Gun survivability is, in fact, a major consideration in perforating system design and manufacture. Achieving reliable survivability requires an understanding of the loading regime and damage mechanisms involved, and the requisite material properties and geometric characteristics. This event bridges hypervelocity impact, blast loading, shock physics, and material science disciplines, as well as rigorous manufacturing process control.

In this paper, we address some of these salient issues in general, and present experimental, analytical, and numerical investigations into perforating gun damage. Subsequent papers will explore the constituent facets in greater detail. Keywords: perforating, gun damage, oilwell, gun splitting, fragment impact.
\end{abstract}




\section{Introduction}

\subsection{Background}

A common method of extracting oil or gas from a subterranean formation begins with drilling a wellbore to intersect the reservoir, lining the borehole with a steel casing, then cementing the casing in place. The well is then perforated, wherein holes are created through the casing and into the productive formation. Perforating establishes hydraulic communication between the reservoir and the wellbore, allowing, in turn, production uphole through surface facilities.

A perforating gun system is the assembly of shaped explosive charges used to perforate a well. The charges are ballistically connected via explosive detonating cord, typically initiated with a single detonator. The gun is lowered through the wellbore from surface to the vicinity of the target reservoir, via cable, tubing, or other conveyance means. At the prescribed time and location, the charges are detonated; creating the desired perforation tunnels (Figure 1).

The presence of wellbore fluid (and pressure) requires some means of isolating the charges from the wellbore environment. This isolation is generally achieved in one of two ways: (1) by packaging the charges within a carrier tube (a sealed hollow - usually steel - tube), or (2) individually sealing each charge, so that each capsule charge housing is exposed to the wellbore environment. This paper focuses on hollow carrier perforating systems.

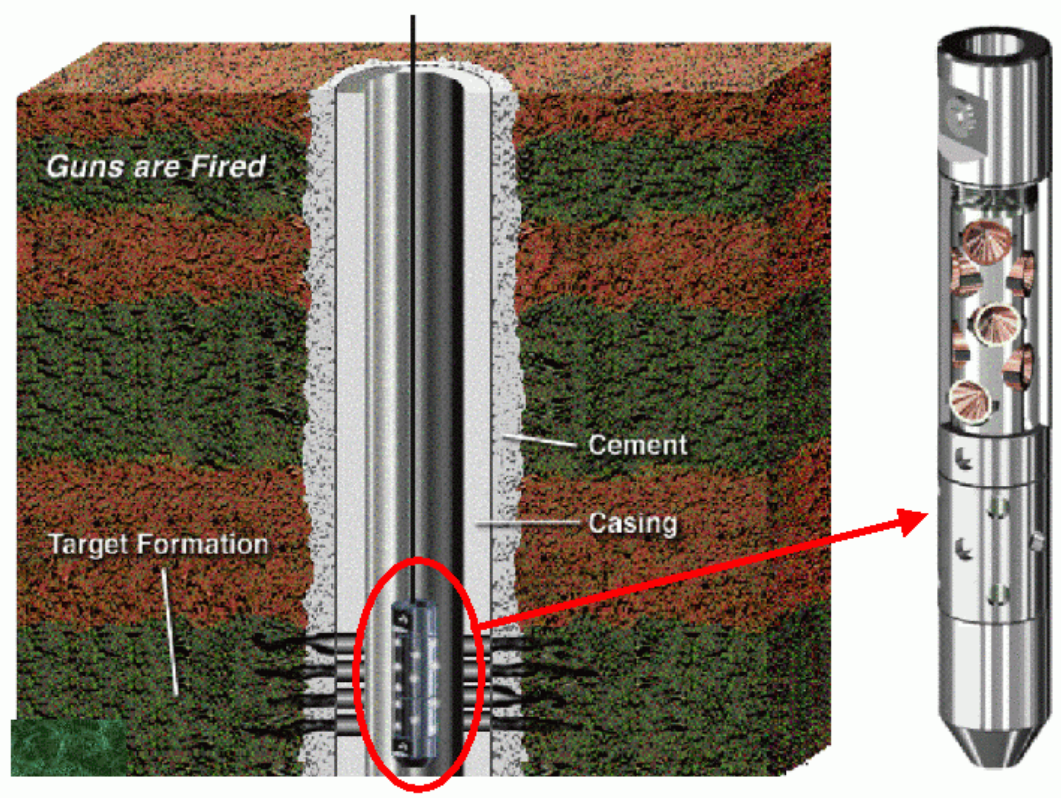

Figure 1: Oilwell perforating schematic. 


\subsection{Motivation}

A perforating gun carrier tube must withstand its environments both before and during perforating. Perforated carriers exhibit varying degrees of swelling, and jet-formed exit holes (the peripheries of which typically contain elevated burrs). These features are considered acceptable damage, provided they remain within certain limits. Extreme forms of damage - excessive swelling, cracking, or catastrophic rupturing (i.e. splitting) - are unacceptable.

Gun survivability is important for several reasons: to ensure reliable retrieval to surface (or dropping off into the rathole - an empty wellbore region below the target formation), to contain perforating debris (i.e. prevent excessive case shrapnel from entering the wellbore), and to isolate much of the undesirable perforating energy from the downhole environment (reservoir rock, completion equipment, etc.).

\section{General considerations}

\subsection{Environment}

Before perforating, the carrier tube must support external pressure (exceeding $20 \mathrm{ksi}$ in some instances) without collapsing or leaking, sometimes at elevated temperature (up to $500^{\circ} \mathrm{F}$ ), and/or in corrosive fluids.

Perforating charge detonation provides a combination of dynamic internal loadings - namely fragment impact and explosive blast, combined with the jet-formed exit holes (which can become stress concentration sites). The fragments which impact the gun ID generally come from the thin steel loading tube, (used to package the charges within the carrier), which is driven by the expanding metallic charge confining cases. For gun designs not employing steel loading tubes, case fragments directly impact the carrier ID.

The interaction of these various internal loadings and the carrier tube (whose response is a function of geometry, composition, manufacturing process, and pre-shooting environmental exposure), determine the extent to which the carrier will survive.

\subsection{Gun characteristics}

Collapse pressure is a function of geometry and yield strength. Since gun size is dictated by operational and performance requirements, yield strength and circumferential uniformity are the critical parameters in resisting collapse.

Diametral swell is determined by the dynamic ductility and yield strength (accounting for the effects of strain and rate hardening, thermal softening, and stress state), in response to the internal blast and fragment impact loadings.

Stress corrosion cracking is specific to the particular environment/material couple, and unrelated to explosive energy. Use of susceptible materials for extended periods, in the presence of significant applied or residual stress, should be avoided. 


\subsection{Global mass and volume ratios}

Oilwell perforating shaped charges generally contain heavy metallic (steel or zinc) confining cases. Charge component mass ratios vary widely, but tend to lie in the approximate range of 10:2:1 (case:liner:explosive).

Since larger diameter guns are generally used to accommodate larger charges or higher shot density (more charges per unit gun length), total component volume per unit internal gun volume is relatively constant across a range of gun sizes. For example, solid explosive generally occupies $\sim 5-10 \%$ of the gun volume, charge cases another $\sim 15 \%$. Liners occupy only $\sim 1 \%$, the loading tube occupies some of the remainder, and the balance remains vacant.

\subsection{Global energy partition}

Hydrocode simulations have been used to calculate the energy partition in a typical functioning charge. Of total explosive energy, approximately $40 \%$ and $30 \%$ go into the kinetic energies of the case and jet, respectively. Most of the balance $(\sim 25 \%)$ remains in the detonation gas (blast energy), while the remaining $5 \%$ goes into shock heating the case and liner.

Since only the jet energy exits the gun, approximately $70 \%$ of the total explosive energy remains in the gun. Much of this is imparted to the gun wall via localized fragment impact, subsequently converted to plastic deformation and heat. All case kinetic energy is not deposited directly into the gunwall, however, since some fragments impact those from adjacent charges; "stopping" each other and heating the adjacent detonation gas (particularly with 3-per-plane systems).

Superimposed upon the non-uniform fragment impact field is explosive blast energy. While the initial blast pressure transients can be localized (including mach stem effects, etc.), later-time ( $\mathrm{msec}$ and beyond) overpressure is more spatially uniform. This "equilibrium" pressure tends to decay due to cooling, which complicates any analysis of a sustained (i.e. static) internal loading.

These characteristic quantities (volume ratios, energy partitions, etc.) are relevant to qualitative considerations. However, given the localized nature of fragment impact, and the time-varying behavior of the detonation gas, it is impossible to develop general predictive analytical gun survivability models based solely on these characteristic global quantities.

\section{Damage mechanisms}

\subsection{Fragment impact}

Charge case expansion velocity can be determined by numerical, analytical, or experimental methods. Velocities typically range from $\sim 300$ to $800 \mathrm{~m} / \mathrm{sec}$, and can vary along the length a given charge, as the local case / explosive thickness ratio can vary significantly. 


\subsubsection{Experimental observations}

Fragment impact is generally the primary gun damage mechanism in continuous phased perforating guns. In these systems, charges are positioned every 1-3" along the gun's length. Fragments impact the gun (via the loading tube), producing localized bulges in the carrier. This can be moderate (Figure 2), or excessive, even leading to unacceptable cracking (Figure 3).

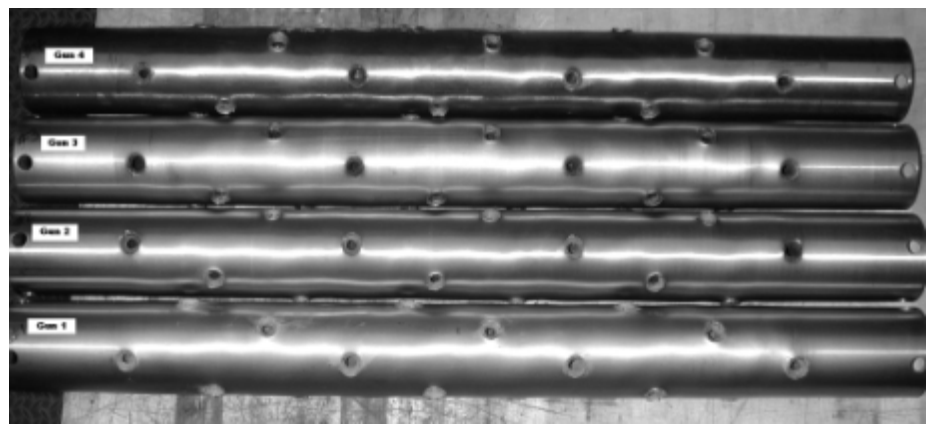

Figure 2: "Survived" perforated guns.

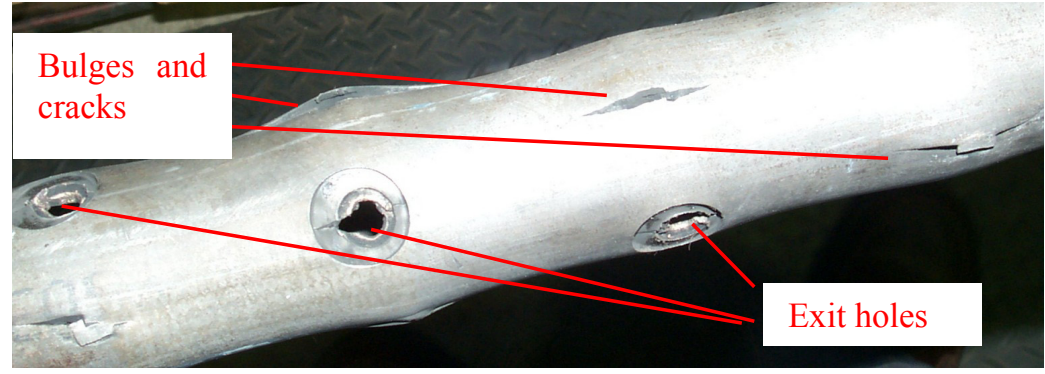

Figure 3: $\quad$ Perforated gun cracked from fragment impact.
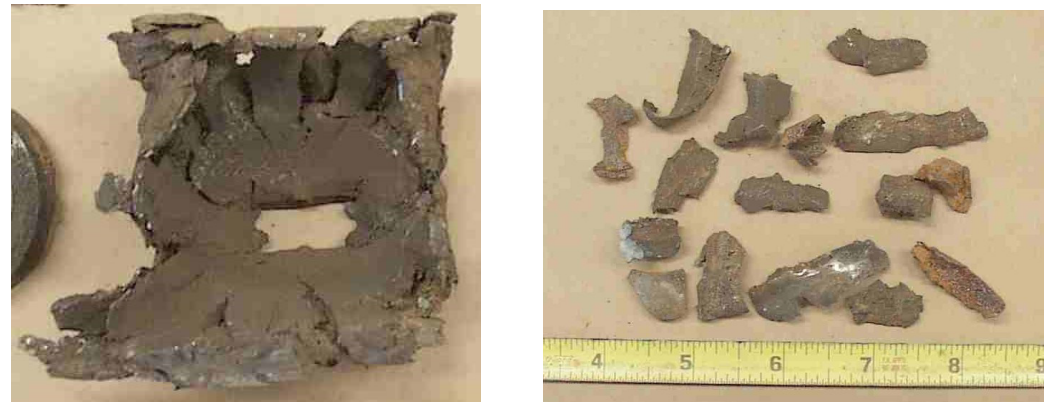

Figure 4: $\quad$ Perforating charge fragments.

Case fragments themselves can cover a broad range of sizes and shapes (discussed further in a subsequent section). The left photo in Figure 4 shows one 
bulky fragment, while the right photo shows typical smaller fragments (some of which result from spall).

\subsubsection{Numerical studies}

Numerical simulations can provide insight into potential stress states and magnitudes seen by the gun carrier, which can then be evaluated against its strength and fracture characteristics.

Figures $5-7$ show results of a computer simulation of a single functioning charge. Due to rotational symmetry about the charge axis, this simulation is of a spherical gun section. However, these results do provide some insights into characteristic velocities and stress profiles in a real cylindrical gun. For this calculation, data-recording tracers were placed throughout the case, loading tube, and gun carrier wall.
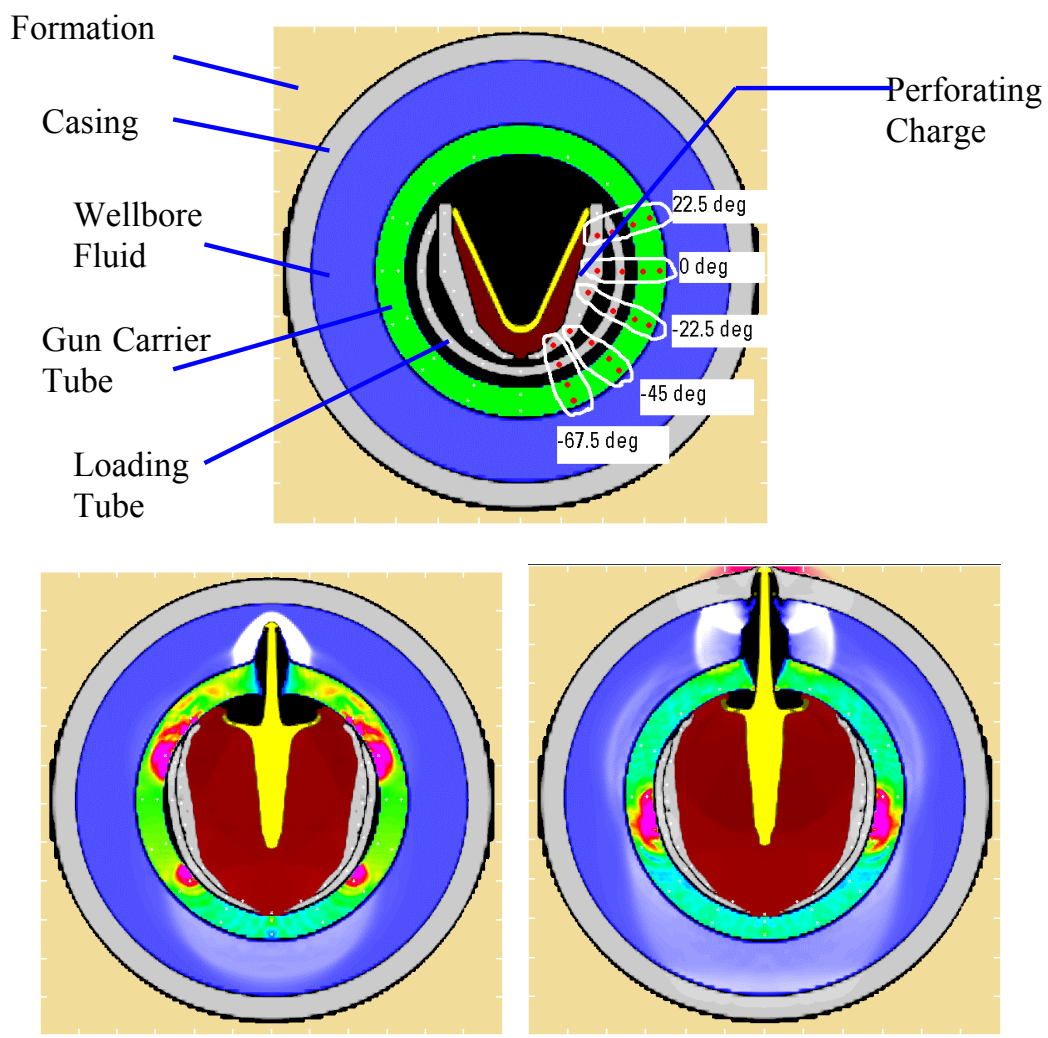

Figure 5: Hydrocode simulation of oilwell perforating charge.

Figure 5 shows snapshots of the functioning charge at various times. These frames reveal many interesting phenomena, but we focus here on the pressure waves within the gun carrier. The second frame shows two shock regions $\left(22.5^{\circ}\right.$ and $-45^{\circ}$ ), where the forward and aft regions of the [case + loading tube] have 
impacted the gun wall, but the middle section has not yet. These shocks travel toward each other, and meet at just about the time that the case mid-section impacts the gun wall, producing a strong shock at $\sim 0^{\circ}$ (third frame).

Velocity: Figure 6 shows velocity-time histories for two selected regions. Both show the case expansion velocity, reduced [case + loading tube] velocity, and resultant gun wall velocity (which decelerates to zero due to gun strength).

The first frame shows a case region whose terminal velocity is $\sim 580 \mathrm{~m} / \mathrm{sec}$, subsequently decelerated to $\sim 380 \mathrm{~m} / \mathrm{sec}$ upon impact with the loading tube.

The second frame shows analogous velocities for a different region. Nearer the charge aft, this case region is thinner, and continues to accelerate through $800 \mathrm{~m} / \mathrm{sec}$ as it impacts the loading tube. The [case + loading tube] impacts the gun wall at $\sim 530 \mathrm{~m} / \mathrm{sec}$, about $40 \%$ faster than the region depicted in the first frame.
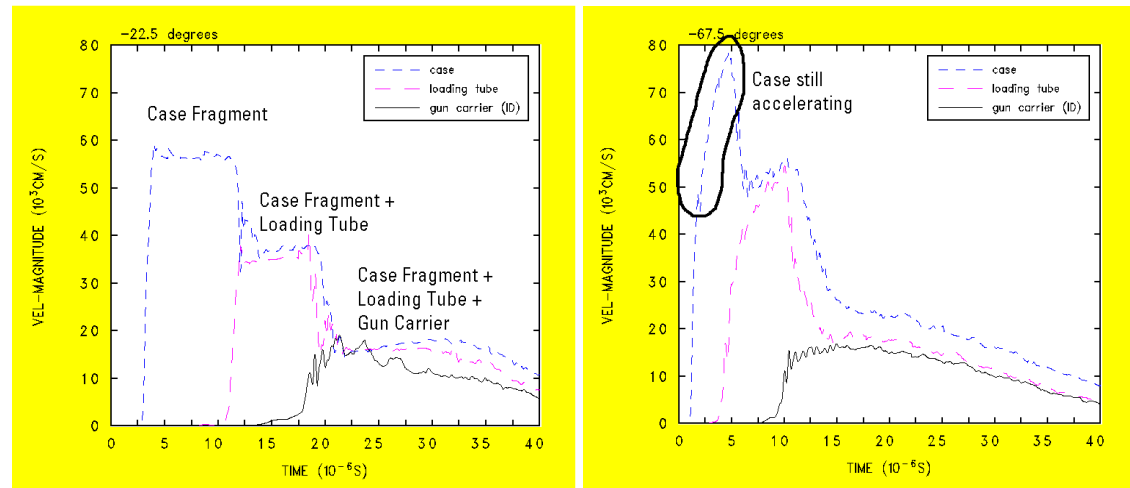

Figure 6: Hydrocode calculated velocities.
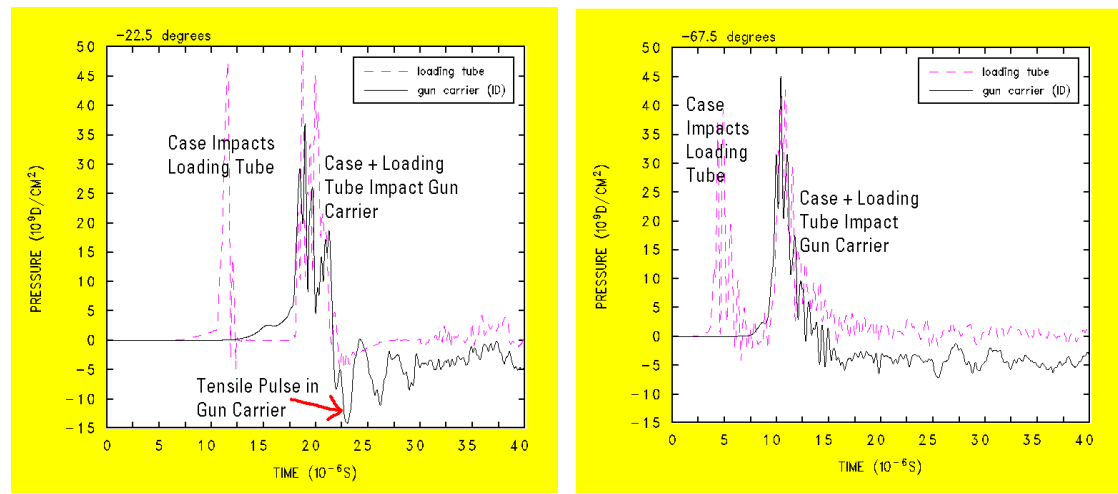

Figure 7: Hydrocode calculated pressures.

Pressure: Figure 7 shows pressure-time histories for the loading tube and gun carrier, at the same two regions. While the second frame reveals a stronger compressive pulse (consistent with higher impact velocity mentioned above), the 
first frame reveals a strong tensile pulse $(\sim 15 \mathrm{kbar})$; this may be important when considering the gun material's ductility in the context of stress state triaxiality.

\subsubsection{Analytical treatment}

Fragment Velocity: Average case fragment velocity may be estimated from the energy partition discussed previously. For example, consider an explosive charge with a $150 \mathrm{~g}$ case, $15 \mathrm{~g} \mathrm{HMX}$ (assuming $5.5 \mathrm{~kJ} / \mathrm{g}$ detonation energy), where $40 \%$ of the explosive energy converts to case kinetic energy. This gives:

$$
\begin{aligned}
V_{\text {case_mean }} & =\sqrt{\frac{2\left(K E_{\text {case }}\right)}{m_{\text {case }}}} \\
& =\sqrt{\frac{2(0.4 \times \exp \text { losive_energy })}{m_{\text {case }}}} \\
& =660 \mathrm{~m} / \mathrm{sec}
\end{aligned}
$$

This is consistent with experimental and numerical values, keeping in mind that true local case velocities can vary significantly above and below this value depending on the local explosive-to-case thickness (i.e. mass) ratios.

Fragment size distribution: A perforating charge is essentially a fragmentation warhead, for which analytical treatments are well established (see Held [1]). Fragmentation characteristics, namely quantity and mass distributions, can be estimated by a number of methods (for example Mott and Linfoot [2], Mott [3,4], Grady and Kipp [5]). Due to space constraints, a detailed analytical treatment of perforating charge case fragmentation will be withheld from the present discussion.

\subsubsection{Carrier response}

Carrier response to fragment impact loading depends strongly on its mechanical properties. The operative loading regimes are comparable to those in traditional light armor applications (though the geometry is different).

The applied stress magnitude, state and loading rate, along with the carrier's strength (including effects of strain and rate hardening, and thermal softening), determine the extent to which the carrier swells, and whether or not it splits.

The carrier wall is subjected to radial compression from external wellbore pressure (static) and internal detonation gas (dynamic, static); longitudinal tension (static) from the weight of the suspended gun string, and internal gas pressure (quasi-static); and hoop tension from detonation gas (dynamic). The ID experiences localized fragment impact, imparting dynamic radial compression, in some instances followed by dynamic tension (rarefaction); accompanying and surrounded by shear.

Compressive stress increases ductility; tensile stress decreases it. Although some diametral growth can be attributed to detonation gas pressure, most swell results from localized bulging at case fragment impacts. 
Figure 8 shows examples of different modes of gun failure. The top photo shows a "window" punched out by charge case fragment impact. The fracture is direct shear; the leading edge probably exhibits shear banding. The microscopic mode is ductile (microvoid coalescence - dimples elongated in the shear direction).
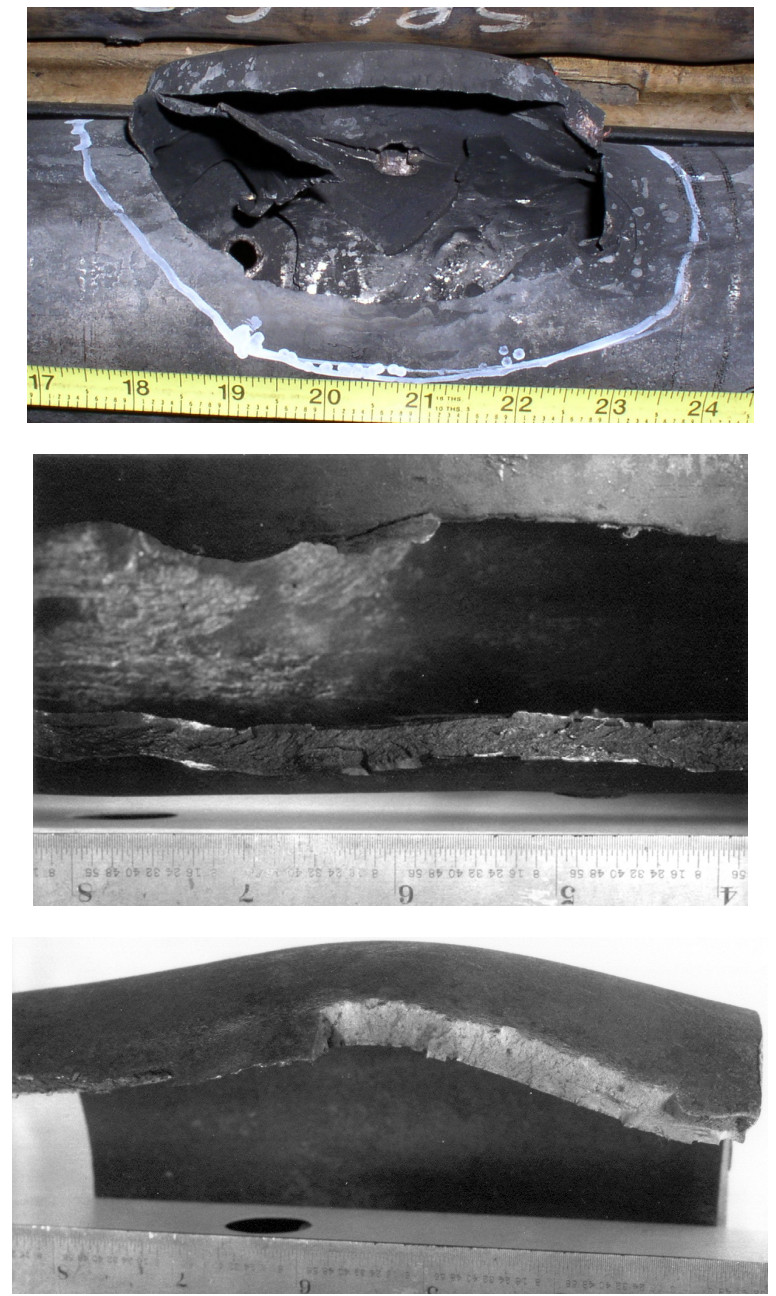

Figure 8: Various gun failure modes

The middle photo shows a fracture initiated at a fragment impact and propagated in a brittle manner, driven by momentum and internal gas pressure. The driving stress was hoop. Microscopically, the fracture mode is cleavage. This steel did not receive satisfactory heat treatment. 
The bottom photo shows a tube sample split in hoop by detonating explosive (coupled to the steel by water) along the longitudinal axis. Fracture is slant-ductile overload, with microvoids elongated in the shear direction. The entire sample is grossly distorted but did not fail in a brittle manner. Steel can be ranked by the amount of explosive required to fail it in this manner, and whether the fracture is "slant" ductile or "flat" brittle.

Carrier response also depends on environmental factors such as wellbore fluid, pressure, and temperature. Perforating guns are fired either in a (liquid) completion fluid, or in a dry wellbore. To address these in-situ environments, researchers qualify guns on surface in either water or air. Table 1 shows typical perforating gun swell diameters. The difference in swell OD (water vs. air) ranges from $\sim 0.03-0.2 "$. In some cases, this difference can make one gun acceptable in a liquid environment, yet unacceptable in a gas environment.

Table 1: $\quad$ Swell dimension of typical perforating guns.

\begin{tabular}{|l|l|l|l|}
\hline No. & $\begin{array}{l}\text { Gun Size, OD } \\
\text { (in.) }\end{array}$ & $\begin{array}{l}\text { Swell OD in } \\
\text { Water (in.) }\end{array}$ & $\begin{array}{l}\text { Swell OD in } \\
\text { Air (in.) }\end{array}$ \\
\hline 1 & 1.56 & 1.72 & 1.75 \\
\hline 2 & 2.00 & 2.16 & 2.21 \\
\hline 3 & 2.50 & 2.59 & 2.75 \\
\hline 4 & 3.38 & 3.56 & 3.77 \\
\hline 5 & 3.67 & 3.96 & 4.01 \\
\hline
\end{tabular}

Downhole temperature (which can be as high as $\sim 500^{\circ} \mathrm{F}$ ) can affect gun survivability in competing ways: elevated temperatures reduce carrier strength, but increase fracture toughness.

\subsection{Explosive blast}

\subsubsection{Experimental observations}

Explosive blast is the major gun damage mechanism in 3-charge-per-plane systems. Here, three charges are positioned within a plane perpendicular to the gun axis, aimed $120^{\circ}$ apart. Alternating planes are rotated $60^{\circ}$, achieving a very high packing density. This "nesting" produces high explosive mass per unit gun volume, a high incidence of case-to-case impact, and minimal case-to-gun impact. If the internal gas pressure is high enough, longitudinal fractures will initiate at the exit holes, where the hoop stress concentration is the highest, and propagate in the longitudinal direction. If a fracture runs from one exit hole to another, catastrophic split can result.

The left picture in Figure 9 shows a 3-charge-per-plane gun after detonation, which "survived" with clean exit holes, and no obvious localized swell or cracks. The right picture shows a failed perforating gun, exhibiting a large fracture running longitudinally from one scallop (exit hole) to another. Shaped charge case fragments can be seen spilling into the crack. 


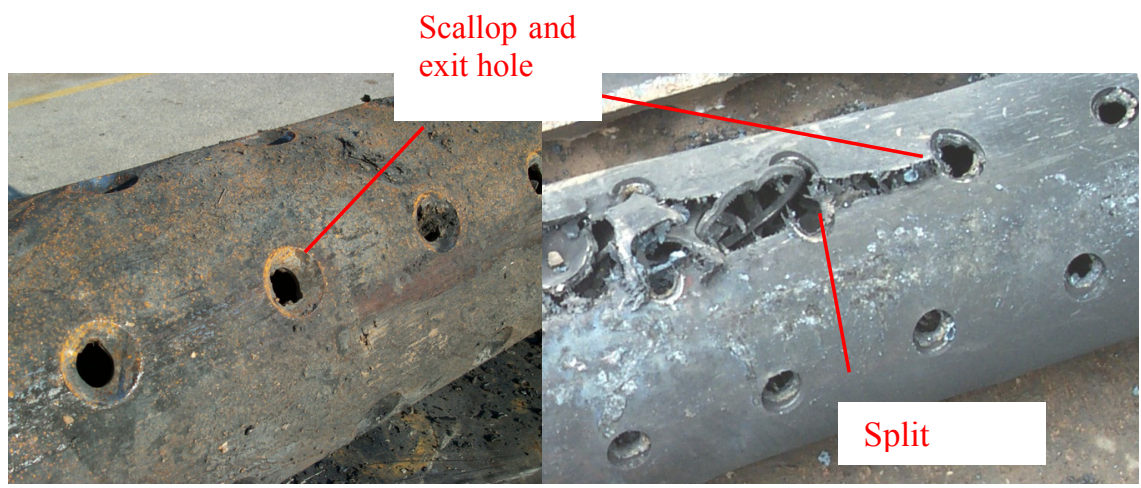

Figure 9: Perforated guns (3-charge-per-plane).

\subsubsection{Numerical studies}

The previously-presented numerical analysis of fragment impact implicitly included the effects of early-time blast loading (albeit for a single charge, approximating a continuous phased system). Numerical investigation of a 3 -per-plane system is much more complicated, due to system geometry, and will therefore not be pursued here.

\subsubsection{Analytical treatment}

While the solid explosive's detonation pressure can be reasonably estimated, this acts on the liner and case, not the gun carrier itself. The pressure exerted against the carrier ID by the detonation product gas is not so easily determined; furthermore, it varies significantly with both position and time.

In the first tens of microseconds after charge initiation, shock waves continue to reflect within the detonation gas, which has expanded to fill the available gun volume. It is believed that these transients are relatively insignificant in comparison to the fragment-induced shock pressures occurring at these early times.

At later times (approaching the millisecond time scale and beyond), gas pressures reach spatial equilibrium within the carrier. At this stage one can begin to estimate characteristic pressures, keeping in mind that these are subject to decay due to cooling and other mechanisms. Since this pressure exerts against the gun interior for a relatively long time (how long of course depends on the decay rate), such pressures can be analyzed within the context of the carrier's static load-bearing capabilities. Due to space constraints, however, a detailed analysis of in-gun gas pressure is withheld from the present discussion.

\section{Summary and conclusion}

We have presented an overview of damage to oilwell perforating guns. Guns are exposed to wellbore fluid which can be liquid or gas, high pressure and/or high temperature, and sometimes corrosive. Shaped explosive charges present three significant and damaging internal loading mechanisms: jet perforation, case fragment impact, and explosive blast. It is important that gun carriers survive 
downhole exposure and perforating, without excessive swelling, cracking, or catastrophic rupturing.

Guns can fail when subjected to an unfavorable combination of geometric/mechanical properties and internal loading. Improper carrier tube metallurgy, heat treatment, etc., and/or excessive localized or sustained internal loading (functions of charge design, manufacture, and packaging) can lead to gun failure. Fragment impact is usually the principal damage mechanism in continuous phased guns, and explosive blast is the primary load in 3-per-plane systems.

Achieving reliable survivability requires an understanding of the loading regime and damage mechanisms involved, and the requisite material properties and geometric characteristics. Perforating and the related issue of gun survivability bridge hypervelocity impact, blast loading, shock physics, and material science disciplines, as well as rigorous manufacturing process control.

We have cursorily addressed fragment impact and blast loading from experimental, analytical, and numerical perspectives; and briefly discussed gun material response to these various loadings. Subsequent papers will explore these issues in greater detail.

\section{References}

[1] Held, M., Fragmentation Warheads (Chapter 8). Tactical Missile Warheads, ed. J. Carleone, AIAA, pp. 387-464, 1993.

[2] Mott, N.F. \& Linfoot, E.H., Ministry of Supply, AC 3348, January, 1943

[3] Mott, N.F., Ministry of Supply, AC 3642, March 1943

[4] Mott, N.F., Ministry of Supply, AC 4035, May, 1943

[5] Grady, D.E. \& Kipp, M.E., Geometric statistics and dynamic fragmentation. Journal of Applied Physics, 58, pp. 1210-1222, 1985 